\title{
Chaotic signals denoising using empirical mode decomposition inspired by multivariate denoising
}

Fadhil Sahib Hasan

Department of Electrical Engineering, Mustansiriyah University, Iraq

\begin{tabular}{l}
\hline \hline Article Info \\
\hline Article history: \\
Received Jun 4, 2019 \\
Revised Oct 17, 2019 \\
Accepted Oct 27, 2019 \\
\hline
\end{tabular}

Keywords:

Chaotic signal

Empirical mode decomposition Multivariate denoising

Wavelet denoising

\begin{abstract}
Empirical mode decomposition (EMD) is an effective noise reduction method to enhance the noisy chaotic signal over additive noise. In this paper, the intrinsic mode functions (IMFs) generated by EMD are thresholded using multivariate denoising. Multivariate denoising is multivariable denosing algorithm that is combined wavelet transform and principal component analysis to denoise multivariate signals in adaptive way. The proposed method is compared at a various signal to noise ratios (SNRs) with different techniques and different types of noise. Also, scale dependent Lyapunov exponent (SDLE) is used to test the behavior of the denoised chaotic signal comparing with clean signal. The results show that EMD-MD method has the best root mean square error (RMSE) and signal to noise ratio gain (SNRG) comparing with the conventional methods.
\end{abstract}

Copyright $\odot 2020$ Institute of Advanced Engineering and Science. All rights reserved.

\section{Corresponding Author:}

Fadhil Sahib Hasan,

Department of Electrical Engineering,

Mustansiriryah University, Iraq.

Email: fadel_sahib@uomustansiriyah.edu.iq

\section{INTRODUCTION}

Chaotic signals have many properties such as aperiodicity, sensitivity to initial conditions, and wideband spectrum that make them suitable to use in many applications areas such as secure communication [1], image encryption [2], speech encryption [3] and other applications. However, when the chaotic signals corrupted with a noise become very hard to find Lyapunove exponent, correlation dimension, Kolmogorov entroy and other chaotic system parameters [4]. Therefore, removes the noise from the chaotic signals in affective way are the main challenges and the great significant in this research.

In the last years, different techniques are introduced to remove the noise from the chaotic signals. The most famouse method using wavelet transform [5-7] in which the chaotic signal is denoised by decomposing it into detail and approximate components and then the details are smoothed using adaptive thresholds. To find optimum threshold for each scale in wavelet domain, genetic algorithm is suggeseted in [8]. In [9-11] dual wavelet transform are used as an extension to wavelet trsform to remove the noise from chaotic signal where optimal decomposition scale and adaptive selecting wavelet coefficients are determined. While wavelet transform contains only time domain locality, synchrosqueezed wavelet transform (SWT) conatins coth time and frequency properties is used with hierarchical threshold to enhance the chaotic signal [12]. Another most famouse denoising technique is empirical mode decomposition (EMD) [13] in which the signal is decomposed into many signals of amplitude and frequency modulated with zero mean value that are called intrinsic mode functions (IMFs) and then at certain threshold select which mode is used to reconstruct the denoised signal. EMD is improved in [14, 15] by using ensemble empirical mode decomposition (EEMD) and EEMD and singular value decomposition (SVD) respectively. Another impvemnt to EMD is depicted in [4] in which zero-crossing scale thrsholding enhancement algorithm is used to enhance noisy chaotic signal. Other denoising chaotgic techniques which are combined EMD and independent component analysis (ICA) are depicted in [16, 17]. 
In this paper, a new denoising technique that is combined both EMD and multivariate denoising using wavelet and principal component analysis (MD-WPCA) to denoise the chaotic signal corrupted by certain additive noise is proposed and named EMD-MD algorithm. MD-WPCA is an extension of wavelet denoising to multivariate signals that is proposed in [18] to denoise multivariate signals instead of univariate signal and it is combined wavelet transform and principal component analysis (PCA). In this paper, inspired by MD-WPCA, an intrinsic mode functions (IMFs) generated by EMD are properly adapted and thresholded to denoise the chaotic signal. Furthermore, scale dependent Lyapunov exponent (SDLE) function is used as a measure to find the amount of enhancement factor for the proposed system comparing to the clean chaotic signal.

The rest of this paper is followed as: Section 2 provides the block diagram of the suggested algorithm. Section 3 provides performance evaluation of noise reduction method. The simulation results of noise reduction are summerized in Section 4. Furthermore, Section 5 contains the conclusion.

\section{EMD BASED CHAOTIC DENOISING INSPIRED BY MULTIVARIATE DENOISING (EMD-MD)}

Figure 1 show the block diagram of EMD based chaotic denoising inspired by multivariate denoising (EMD-MD). In this system, the clean chaotic signal $x(n)$ is corrupted by a noise $w(n)$ with length $\mathrm{N}$, then the noisy chaotic signal $r(n)$ is given by:

$$
r(n)=x(n)+w(n), n=1, \ldots, N
$$

The objective is to separate the clean chaotic signal from the noise signal and recover the interest clean chaotic signal. In the first step, the signal $x(n)$ is decomposed into a set of $L$ basis function called intrinsic mode functions (IMFs), $c_{i}(n), i=1, \ldots, \mathrm{L}$, using EMD algorithm $[4,13,14]$. Two conditions are required in each IMFs $[13,14]$ : First, the extrema number and zero crossing number must be equal or differ at most by one. Second, the average value of the upper and lower envelopes defined by the local maxima and minima must be zero. One of the most famous algorithms to find each IMFs is called sifting process that is iterative process. The procedure of sifting algorithm can be summerized in briefly as $[4,13,14]$ :

1) Compute local maxima, $\max _{\mathrm{j}}, \mathrm{j}=1,2, \ldots$ and local minima, $\min _{\mathrm{k}}, \mathrm{k}=1,2, \ldots$ in $r(\mathrm{n})$.

2) Using cubic spline interpolation to construct the upper and lower envelope, $\max (\mathrm{n})=f_{\max }(\max , n)$ and $\min (\mathrm{n})=f_{\min }\left(\min _{k}, n\right)$ respectively.

3) Find the envelope mean, $e(n)=[\max (n)+\min (n)] / 2$.

4) If $\mathrm{e}(\mathrm{n})$ satisfies the IMF conditions, assign $c_{i}(n)=e(n)$ for $i$ th IMF and update $r(\mathrm{n})$ as

$$
r(n)=r(n)-c_{i}(n)
$$

5) If $r(n)$ remains approximately unchanged then back to step (1) and stop.

6) After obtaining an IMFs, $c_{i}(n)$, subtract $c_{i}(n)$ from the signal $\mathrm{r}(\mathrm{n})=\mathrm{r}(\mathrm{n})-c_{i}(n)$ and back to step (1) if $\mathrm{r}(\mathrm{n})$ is not constant or trend the residual signal, $\rho(\mathrm{n})$.

Consequently, the original signal, $r(n)$, is recovered by the following equation:

$$
r(n)=\sum_{i=1}^{L} c_{i}(n)+\rho(n)
$$

In the next step, the IMFs signals are passing through MD-WPCA algorithm to get the denoised version of the IMFs signals. MD-WPCA is proposed by Aminghafari [18] to remove noise from multivariate noisy signals by combined principal component analysis (PCA) and univariate wavelet thresholding. Given the IMFs signals from the previous step, $c_{i}(n)$, and the residual $\rho(n)$ and denoted by C(i) where C(i) is the matrix form of $c_{i}(n), \mathrm{C}(\mathrm{i}) \in N \times(L+1)$. The MD-WPCA algorithm is outlined in the following steps:

1) Apply the DWT at a level $\mathrm{J}$ for each column of $\mathrm{C}$ to obtain the $(\mathrm{J}+1)$ detail coefficients matrices $\mathrm{D}_{\mathrm{j}}, \mathrm{j}=1, \ldots, \mathrm{J}$ at level 1 to $\mathrm{J}$ and the approximate coefficients $\mathrm{A}_{\mathbf{J}}$ of $\mathrm{L}+1$ channels, where $\mathrm{D}_{\mathrm{j}} \in N 2^{-j} \times(L+1), \mathrm{j}=1, . ., \mathrm{J}$ matrices and $\mathrm{A}_{\mathrm{J}} \in N 2^{-J} \times(L+1)$ matrix.

2) Find the noise covariance estimate $\sum_{c}$ by applying the minimum covariance determinant (MCD) to $\mathrm{D}_{1}\left(\sum_{c}=\mathrm{MCD}\left(\mathrm{D}_{1}\right)\right)$. Then find an orthogonal matrix $\mathrm{V}$ by computing the singular value decomposition (SVD) of $\sum_{c}\left(\sum_{c}=V \Lambda V^{T}\right)$, where $\Lambda=\operatorname{diag}\left(\lambda_{i}, \mathrm{i}=1, \ldots, L+1\right)$ and $\lambda_{i}, \mathrm{i}=1, \ldots, \mathrm{L}+1$ are the eigenvalues for each channel.

3) Next, change the basis using $V$ for each detail $D_{j}$ by using the following multiplication, $E_{j}=D_{j} V, j=1, . ., J$, and apply the universal threshold $t_{i}=\sqrt{2 \lambda_{i} \log (N)}, \mathrm{i}=1, \ldots, \mathrm{L}+1$ for the $i$ th column of $\mathrm{E}_{\mathrm{j}}$ to obtain $\hat{E}_{j}$.

4) Find the PCA of the matrix $A_{J}$ and select the suitable number $L_{J+1}$ of useful principal component. 
5) Change the basis of $\hat{E}_{j}$ using $V^{T}$ and then make an inverse DWT to obtain the enhanced multivariate signals $\tilde{c}_{i}(n)$.

6) Apply PCA to $\tilde{c}_{i}(n)$ and return the most significant principal components.

The final step, the denoised chaotic signal $\tilde{x}(n)$ is recovered from $\tilde{c}_{i}(n)$ according to:

$$
\tilde{x}(n)=\sum_{i=1}^{L+1} \tilde{c}_{i}(n), n=1, \ldots, N .
$$

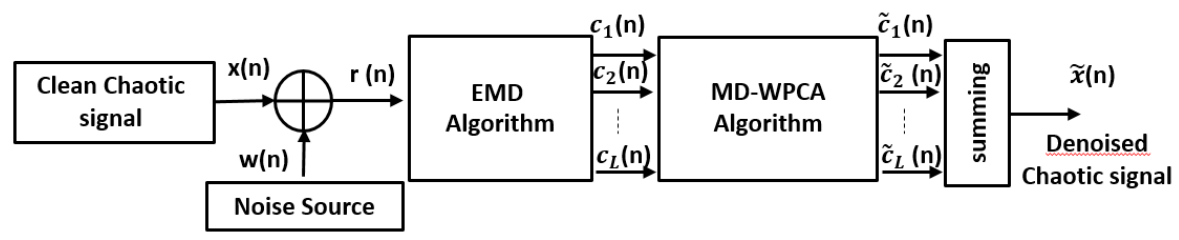

Figure 1. The proposed chaotic denoising system

\section{PERFORMANCE EVALUATION OF NOISE REDUCTION METHOD}

Let us defined $x(n)$ and $\tilde{x}(n)$ as the clean and denoised chaotic signal respectively. In order to compare between the different noise reduction methods, there are different formulas that are used as a performance evaluation measurement such as signal to noise ratio (SNR) [9, 10], root mean square error (RMSE) $[6,8,10]$ and signal to noise ratio gain (SNRG) $[8,10]$. The formulas of these measures are defined as:

$$
\begin{aligned}
& S N R=10 \times \log _{10}\left[\frac{\operatorname{var}(x(n))}{\operatorname{var}(\tilde{x}(n)-x(n))}\right] \\
& R M S E=\sqrt{\frac{1}{2 N} \sum_{n=1}^{N}(x(n)-\tilde{x}(n))^{2}} \\
& S N R G=S N R-S N R_{i}
\end{aligned}
$$

where $\operatorname{var}(x(n))$ is the variance of clean chaotic signal, $\operatorname{var}(\tilde{x}(n)-x(n))$ is the variance of the error between clean and denoised chaotic signal that is equivalent to the noise and $\mathrm{SNR}_{\mathrm{i}}$ is the input signal to noise ratio that is considered in the range $(0-30) \mathrm{dB}$ with step about $5 \mathrm{~dB}$.

Other measure that help us to know whether the noisy chaotic signal is perfectly denoised or not is the scale dependent Lyapunov exponent (SDLE) [19,20]. The algorithm of SDLE is summarized in algorithm 1 .

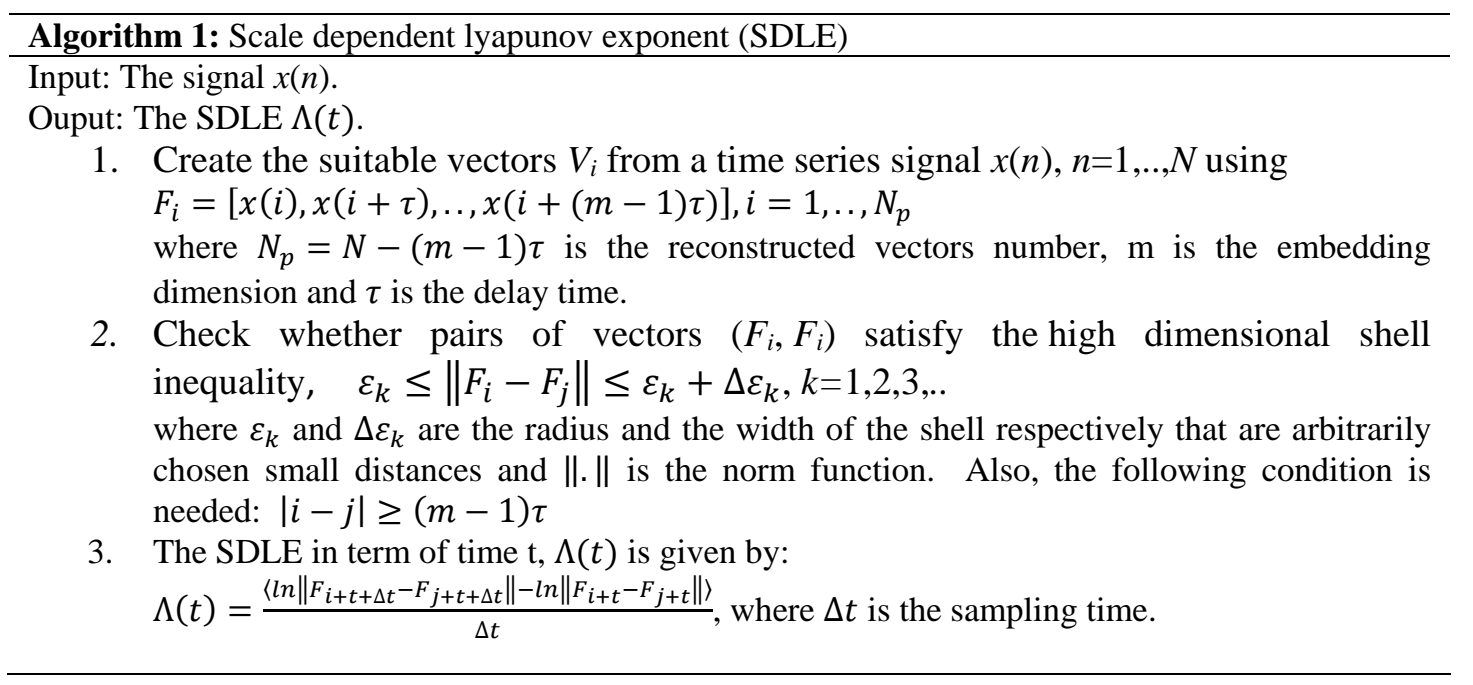




\section{SIMULATION RESULTS}

In this simulation, Lorenz [5], Chen [21] and Rossler [22] are used as chaotic systems to test the proposed method. The chaotic system equations of Lorenz, Chen and Rossler with their setting parameters are described in (7), (8) and (9) respectively:

Lorenz system [5]:

$$
\begin{array}{ll}
d x / d t & =\sigma(y-x) \\
d y / d t & =x(\alpha-z)-y \\
d z / d t & =x y-\beta z
\end{array} \quad, \sigma=10, \alpha=28, \beta=8 / 3 .
$$

Chen system [21]:

$$
\begin{array}{ll}
d x / d t=a(y-x) & \\
d y / d t=x(c-a)-y & , \mathrm{a}=35, \mathrm{~b}=3 \text { and } \mathrm{c}=28 \\
d z / d t=x y-b z &
\end{array}
$$

Rossler system [22]:

$$
\begin{aligned}
d x / d t & =-y-z & \\
d y / d t & =x+a y & , \mathrm{a}=0.38, \mathrm{~b}=0.3 \text { and } \mathrm{c}=4.82 \\
d z / d t & =b x-c z+x z &
\end{aligned}
$$

The differential equations of these systems are solved using a $4^{\text {th }}$ order Runge-Kutta method with a step size of $0.001 \mathrm{sec}$ with 50000 numbers of samples. The different simulation scenarios are depicted below.

Figure 2 and Figure 3 show SNRG and RMSE tests of EMD-MD method respectively to remove AWGN in Lorez, Chen and Rossler chaotic system. The performance evaluation are applied to only $\mathrm{x}(\mathrm{n})$ signal of these chaotic systems. The range of SNRi is $(0-30 \mathrm{~dB})$ with step $5 \mathrm{~dB}$. From these two figures, it can be noticed that SNRG for all types of chaotic systems has at least $17 \mathrm{~dB}$ gain over unenhanced system. Also, Lorenz system has the lowest RMSE values compared with Chen and Rossler for different SNRi.

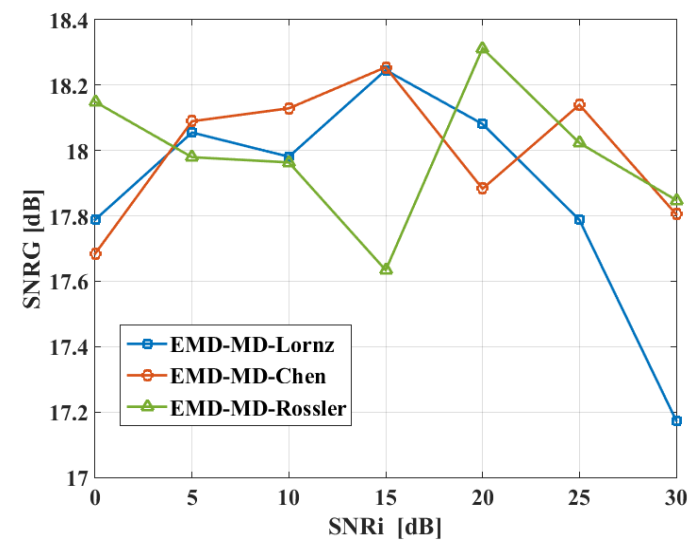

Figure 2. The SNRG measures for different types of chaotic systems when EMD-MD algorithm and AWGN are used

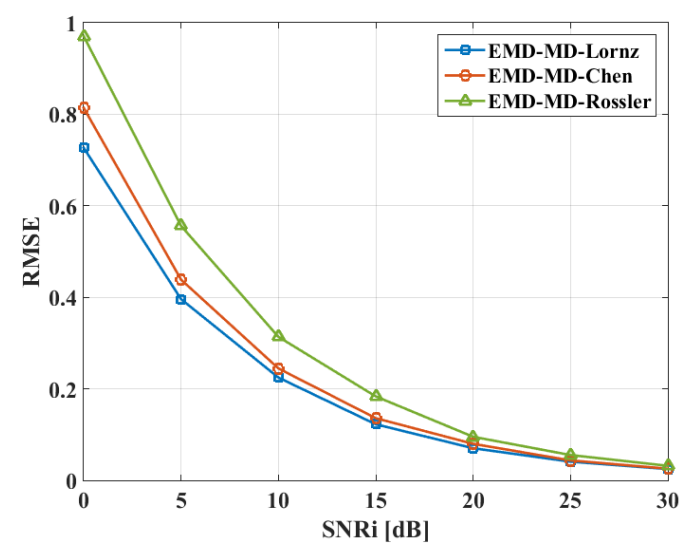

Figure 3. The RMSE measure for different types of chaotic systems when EMD-MD algorithm and AWGN are used

In this simulation, different types of noise are used to test the ability of the proposed system to remove noise. The selected additive noises are AWGN, Factory, Babble, Pink and HFchannel noise that are extracted from Noisex-92 database [23]. The range of SNRi is $(0-30 \mathrm{~dB})$ with step $5 \mathrm{~dB}$. Figure 4 and Figure 5 show SNRG and RMSE tests of EMD-MD method respectively to remove noise in Lorenz chaotic system with different types of noise (AWGN, Factory, Babble, Pink and HFchannel). It can be seen that from these figures, Factory noise has the worst SNRG performance about $4 \mathrm{~dB}$ and the worst RMSE performance compared with other noises. AWGN and Pink noise approximately have the same performance. Also, HFchannel noise has the best SNRG performance about $26 \mathrm{~dB}$ and the best RMSE performance compared with other noises. 


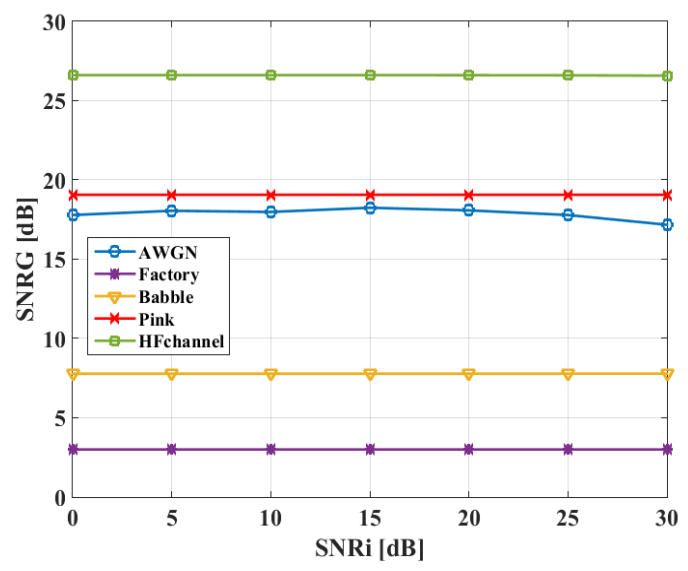

Figure 4. The SNRG measure for different noise types when EMD-MD algorithm and Lorenz chaotic system are used

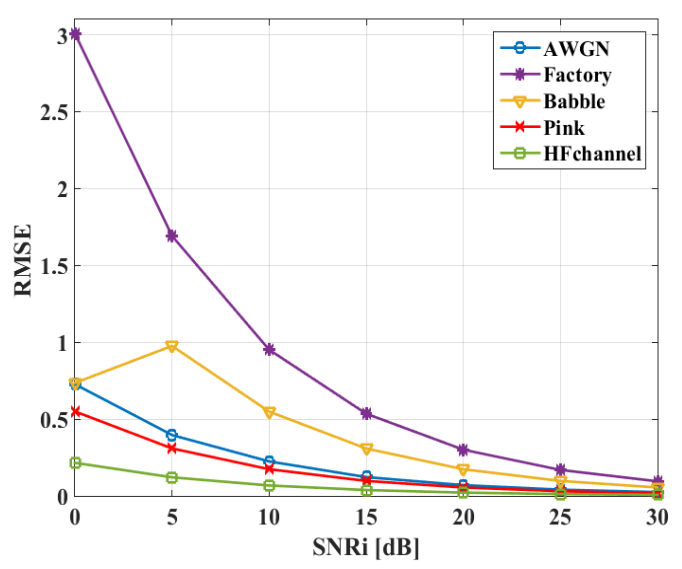

Figure 5. The RMSE measure for different noise types when EMD-MD algorithm is applied to Lorenz chaotic system

Table 1 shows the comparison of the prposed method with different denoised techniques. The parameter of simulation is setting as: the scale $J=4$, wavelet family $=\mathrm{db} 10$ and the threshold is soft threshold, the sampling time $=0.01$. From this table it can be seen that the proposed method has the best SNR and RMSE values comparing with other methods. The SDLE curve for the noise free Lorenz signal and denoised Lorenz signal using EMD-MD technique for $S N R_{i}=(0,5,15,20) \mathrm{dB}$ is shown in Figure 6. Here $\mathrm{m}=5$ and $\tau=4$. From these figures, it can notice that the curve of denoised signal SDLE is go away from the curve of clean signal when $\mathrm{SNR}_{\mathrm{i}}$ is decreased or noise level is increased. Therefore, the SDLE measure gives good estimation about the level of noise in the noisy chaotic signal and distinguish noise from chaos signal.

Table 1. The comparison of the prposed method with different denoised techniques

\begin{tabular}{lccc}
\hline \multicolumn{1}{c}{ Method } & Chaotic signal & SNRi [dB] & SNR [dB] \\
\hline Wavelet soft threshold ( Han et al. 2007) [5] & Lorenz & 14 & 23.18 \\
Dual wavelet and spatial correlation ( Han et al. 2009) [9] & Lorenz & 14 & 0.3840 \\
Adaptive dual-lifting wavelet (Y. Liu and X. Liao 2011) [10] & Lorenz & 14 & 24.6039 \\
Proposed method & Lorenz & 14 & 24.6631 \\
Improved EEMD (X. Wei et al. 2016) [15] & Lorenz & 15 & 25.0361 \\
Proposed method & Lorenz & 15 & 0.319 \\
Improved EMD (M. Wang et al. 2018) [16] & Chen & 15 & 24.732 \\
Proposed method & Chen & 15 & 23.119 \\
\hline
\end{tabular}

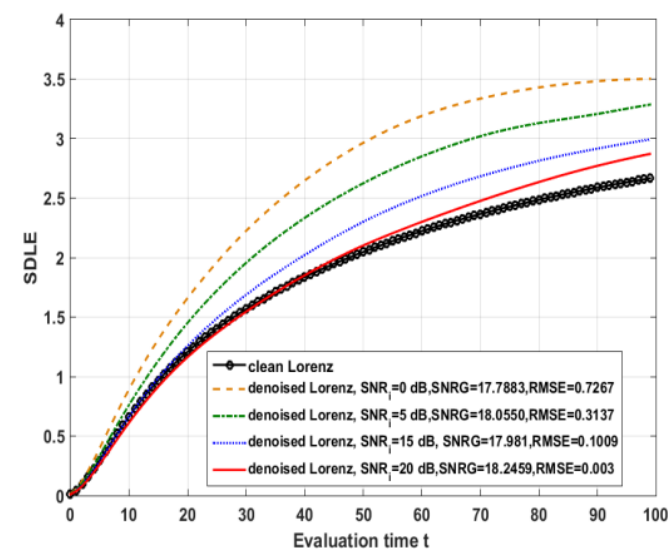

Figure 6. The SDLE curve for the noise free Lorenz signal and denoised Lorenz signal for various values of $\operatorname{SNR}_{\mathrm{i}}(0,5,15,20) \mathrm{dB}$. Here $\mathrm{m}=5, \tau=4$ 


\section{CONCLUSION}

In this paper, the proposed Multivariate Denoising (MD) depends on wavelet and principal component (MD-WPC) thresholded empirical mode decomposition (EMD) based chaotic signal denoising is investigated and named (EMD-MD). In EMD-MD, the MD-WPC is suggested to threshold the intrinsic mode functions (IMFs) of the noisy chaotic signal. The proposed system is tested for different types of chaotic signals, Lorenz, Chen and Rossler system, and different types of noise, AWGN, Factory, Babble, Pink and HFchannel. The prposed method is comparing with conventional chaotic denoising techniques. The results show that EMD-MD has the best SNRG and RMSE values. Furthermore, scale dependent Lyapunov exponent (SDLE) is used to distinguish the evel of noise comparing to the clean chaotic signal.

\section{ACKNOWLEDGEMENTS}

This work is supported by the Faculty of Engineering/ Mustansiriyah University (https://webmail.uomustansiriyah.edu.iq).

\section{REFERENCES}

[1] A. N. Miliou, I. P. Antoniades, S. G. Stavrinides, A. N. Anagnostopoulos, "Secure Communication by Chaotic Synchronization: Robustness under noisy conditions," Nonlinear Analysis: Real World Applications, vol. 8, no. 3, pp. 1003-1012, July 2007.

[2] Chong Fu, Gao-yuan Zhang, Mai Zhu, Zhe Chen, and Wei-min Lei, "A New Chaos-Based Color Image Encryption Scheme with an Efficient Substitution Keystream Generation Strategy," Hindawi Security and Communication Networks, vol. 2018, pp. 1-13, 2018.

[3] Fadhil S. Hasan, "Speech Encryption using Fixed Point Chaos based Stream Cipher (FPC-SC)," Eng. \&Tech. Journal, vol. 34, no. 11, pp. 2152-2166, 2016

[4] M. Wang, Z. Zhou, Z. Li and Y. Zeng, "An adaptive Algorihtm for Chaotic Signals Based on Improved Empirical Mode Decomposition," Circuits, Systems and Signal Processing, vol. 38, no. 6, pp. 2471-2488, 2018.

[5] M. Han, Y. Liu, J. Xi, and W. Guo, "Noise smoothing for nonlinear times series using Wavelet Soft Threshold," IEEE Signal Processing Letters, vol. 14, no. 1, pp. 62-65, 2007.

[6] J. Gao, H. Sultan, J. Hu and Wen-wen Tung, "Denoising Nonlinear Time Series by Adaptive Filtering and Wavelet Shrinkage: A Comparison,” IEEE Signal Processing Letters, vol. 17, no. 3, pp. 237-240, 2010.

[7] Deng Ke, Zhang Lu and Luo Mao-Kang, "A Denoising Algorithm for noisy Chaotic Signals based on the higher order Threshold Function in Wavelet-Packet," Chinese Physics Letters, vol. 28, no. 2, pp. 1-4, 2011.

[8] Xiao Hong Han and Xiao Ming Chang, "Genetic Algorithm Assisted Wavelet noise Reduction Scheme for Chaotic Signals," Journal of Optimization Theory and Applications, vol. 151, no. 3, pp. 646-653, 2011.

[9] M. Han and Y. Liu, "Noise Reduction Method for Chaotic Signals Based On Dual-Wavelet and Spatial Correlation,” Expert Systems with Applications, vol. 36, no. 6, pp. 10060-10067, 2009.

[10] Y. Liu and X. Liao, "Adaptive Chaotic Noise Reduction Method Based on Dual-Lifting Wavelet," Expert Systems with Applications, vol. 38, no. 3, pp. 1346-1355, 2011.

[11] E. Ercelebi, "Electrocardiogram Signals de-noising using Lifting-based Discrete Wavelet Transform," Computers in Biology and Medicine, vol. 34, no. 6, pp. 479-493, 2004.

[12] Wen-Bo Wang, Yun-yu Jing, Yan-chao Zhao, Lian-Hua Zhang and Xiang-Li Wang. "Chaotic Signal Denoising Based on Hierarchical Threshold Synchrosqueezed Wavelet Transform," 1st International Global on Renewable Energy and Development (IGRED), vol. 100, pp. 1-6, 2017.

[13] D. Siwal, V. Suyal, A. Prasad, S. Mandal and R. Singh, "A new Approach of Denoising the Regular and Chaotic Signals using Empirical Mode Decomposition: Comparison and Application," Review of Scientific Instruments, vol. 84, no. 7, pp 1-10, 2013.

[14] M. Wang, Z. Wu, Y. Chen and J. Feng, "Denoising of Chaotic Signals Based on Ensemble Empirical Mode Decomposition," IEEE International Conference on Signal Processing, Communications and Computing (ICSPCC), pp. 14-17, 2014.

[15] X. Wei, R. Lin, S. Liu and C. Zhang, "Improved EEMD Denoising Method Based on Singular Value Decomposition for the Chaotic Signal," Hindawi Publishing Corporation Shock and Vibration, vol. 2016, no. 12, pp. 1-14, 2016.

[16] X. Li and W. Wang, Studying on Denoising of Chaotic Signal using ICA and EMD,"Geo-Informatics in Resource Management and Sustainable Ecosystem, pp. 564-572, 2013.

[17] W. Wang, X. Zhang, Y. Chang, X. Wang, Z. Wang, X. Chen and L. Zheng, "Denoising of Chaotic Signal using Independent Component Analysis and Empirical Mode Decomposition With Circulate Translating," Chin. Phys. B. vol. 25, no. 1, pp. 1-7, 2016.

[18] M. Aminghafari, N. Cheze and J. PoggI, "Multivariate Denoising using Wavelets and Principal Component Analysis,” Computational Statistics \& Data Analysis, vol. 50, no. 9, pp. 2381-2398, 2006. 
[19] J. Gao, J. Hu, W. W. Tung, Y. H. Cao, "Distinguishing chaos from noise by Scale-dependent Lyapunov Exponent," Phys. Rev. E Stat. Nonlin. Soft Matter Phys, vol. 74, no. 6, pp. 1-9, 2006.

[20] J. Gao, J. Hu, W. W. Tung and E, Blasch, "Multiscale Analysis of Biological Data by Scale-dependent Lyapunov Exponent," Front Physiology, vol. 2, pp. 1-13, 2011.

[21] G. Chen, The Chen system revisited, Dynamics of Continuous, Discrete and Impulsive Systems, Series B: Applications \& Algorithms 20, pp. 691-696, 2013.

[22] P. Gaspard, Rossler system, Encyclopedia of Nonlinear Science, pp. 808-811, 2005.

[23] A. Varga and H. J. M. Steeneken, "Assessment for automatic speech recognition ii: Noisex-92: A database and an experiment to study the effect of additive noise on speech recognition systems" Speech Commun, vol. 12, no. 3, pp. 247-251, 1993.

\section{BIOGRAPHY OF AUTHORS}

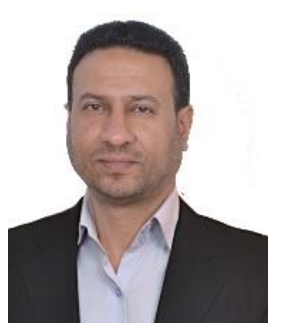

Fadhil S. Hasan was born in Baghdad, Iraq in 1978. He received his B.Sc. degree in Electrical Engineering in 2000 and his M.Sc. degree in Electronics and Communication Engineering in 2003, both from the Mustansiriyah University, Iraq. He received Ph.D. degree in 2013 in Electronics and Communication Engineering from the Basrah University, Iraq. In 2005, he joined the faculty of Engineering at the Mustansiriyah University in Baghdad. His recent research activities are Wireless Communication Systems, Multicarrier System, Wavelet based OFDM, MIMO System, Speech Signal Processing, Chaotic Modulation, FPGA and Xilinx System Generator based Communication System. Now he has been an Assist. Prof. at the Mustansiriyah University, Iraq. Email: fadel_sahib@uomustansiriyah.edu.iq 